\title{
O Uso da Informação de Custos no Setor Público Brasileiro: uma Visão Bibliográfica
}

Simão Pereira da Silva
Mestrado em Administração pela Faculdades Integradas de Pedro Leopoldo - FIPEL
Professor da Universidade Federal dos Vales do Jequitinhonha e Mucuri - UFVJM
Rua do Cruzeiro, no․ 01. Jardim São Paulo. Teofilo Otoni/MG. Brasil. CEP: 39803-371

E-mail:professorsimao@ufvjm.edu.br

Melania Leão Botelho

Bacharelado em Ciências Contábeis pela Universidade Federal dos Vales do Jequitinhonha e Mucuri - UFVJM

Rua do Cruzeiro, no. 01. Jardim São Paulo. Teofilo Otoni/MG. Brasil. CEP: 39803-371

E-mail:melania.botelho@gmail.com

Kenia Fabiana Cota Mendonça

Mestrado em Administração pela Universidade Federal de Viçosa - UFV

Professora da Universidade Federal dos Vales do Jequitinhonha e Mucuri - UFVJM Rua do Cruzeiro, no․ 01. Jardim São Paulo. Teofilo Otoni/MG. Brasil. CEP: 39803-371

E-mail: kenia.mendonca@ufvjm.edu.br

Ronan Pereira Capobiango

Mestrado em Administração pela Universidade Federal de Viçosa - UFV

Professor da Universidade Federal dos Vales do Jequitinhonha e Mucuri - UFVJM Rua do Cruzeiro, no. 01. Jardim São Paulo, Teofilo Otoni/MG. Brasil. CEP: 39803-371 E-mail: ronan.capobiango@ufvjm.edu.br

\section{RESUMO}

Com a padronização da então Contabilidade Pública às normas internacionais de contabilidade do setor público, a implantação do uso da informação de custos no setor público passou a ser discutida como mecanismo de avaliação da eficiência da administração pública. Diante da tempestividade do assunto, o foco desta pesquisa foi analisar como o uso da informação de custos no setor público tem sido debatido no meio acadêmico, visando encontrar evidências de sua aplicação e resultados. Buscouse analisar, por meio das pesquisas constantes dos artigos publicados nos congressos e periódicos nacionais das áreas de Administração e Contabilidade referenciadas pelo qualis/CAPES de 2010 a 2013, a produção acadêmica sobre a implantação do uso da informação de custos no setor público. A metodologia aplicada à pesquisa foi um estudo descritivo através da pesquisa bibliográfica, numa abordagem qualitativa que analisou as informações em relação ao tema. Foram analisados 32 artigos encontrados em periódicos nacionais e 51 artigos encontrados em 5 congressos nacionais. Os resultados revelaram que o debate é recente e que há relevantes contribuições acadêmicas sobre a informação de custos implantada no setor público, principalmente 
O Uso da Informação de Custos no Setor Público Brasileiro: uma Visão Bibliográfica

Simão Pereira da Silva, Melania Leão Botelho, Kenia Fabiana Cota Mendonça, Ronan Pereira

Capobiango

nas áreas da educação, da saúde e da segurança. Os métodos de custeio mais discutidos foram: o ABC e por Absorção com adaptações. Entretanto, há necessidade de preparação das estruturas administrativas para se ampliar a implantação do uso da informação de custos.

Palavras-chave: Informação de Custos. Setor Público. Qualis/CAPES.

\section{The Use of Cost Information in Brazilian Public Sector: A Bibliographical View}

\section{ABSTRACT}

With the standardization of the so-called Public Accounting to international accounting standards to the public sector, the implementation of the use of cost information in the public sector began to be discussed as a mechanism for evaluating the efficiency of public administration. According to the oportunitiy of the subject, the focus of this research was to analyze how the use of cost information in the public sector has been debated in academy, aiming to find evidence of its implementation and results. We attempted to analyze through the constant research of published articles, in national conferences and journals in the areas of Administration and Accounting referenced by qualis/CAPES of 2010-2013, the academic research on the implementation of coast information in the public sector. The applied methodology to the research was a descriptive and exploratory study through bibliographic research, with a qualitative approach that analyzed the information on the subject. Thirty two (32) articles found in national journals and 51 articles found in 5 conferences were analyzed. The results revealed that the debate is recent, and that there are relevant academic contributions on the deployed cost information in the public sector, mainly in the areas of education, health and safety. The most discussed costing methods were $A B C$ and Absorption with adaptations. However, it is necessary to prepare the administrative structures to extend the implementation of the use of cost information.

Keywords: Cost Information. Public sector. Qualis / CAPES.

\section{INTRODUÇÃO}

A administração pública passou por reformas estruturais ao longo dos anos, atravessando várias etapas e chegando a um estágio que preza a eficácia, eficiência e efetividade (MATIAS-PEREIRA, 2012). Neste estágio, promove-se a busca por ferramentas necessárias para um melhor gerenciamento dos gastos públicos. Como área estratégica para a gestão pública, a contabilidade pública deixou de ser voltada 
O Uso da Informação de Custos no Setor Público Brasileiro: uma Visão Bibliográfica Simão Pereira da Silva, Melania Leão Botelho, Kenia Fabiana Cota Mendonça, Ronan Pereira

apenas para o orçamento e passou a dedicar-se mais ao estudo do patrimônio público e suas variações. Tal alteração teve como ponto de partida a Portaria 184, de 25/08/2008, do Ministério da Fazenda, que dispõe sobre as diretrizes a serem observadas no setor público, convergentes às Normas Internacionais de Contabilidade do Setor Público. Em 2011, o CFC (Conselho Federal de Contabilidade) editou as 11 Normas Brasileiras de Contabilidade Aplicadas ao Setor Público (NBCASP), visando orientar a convergência das normas brasileiras de contabilidade do setor público às normas internacionais de contabilidade aplicadas ao setor público.

Destacam-se a Resolução CFC 1.129/08, que aprova a NBCT 16.2 que trata do patrimônio e sistemas contábeis, e a Resolução 1.366/11, que aprovou a NBCT 16.11 (Sistema de Informação de Custos do Setor Público), cuja finalidade foi estabelecer conceituação, objetivo e regras básicas para mensuração e evidenciação dos custos no setor público, para fatos ocorridos a partir de 2012.

Diante dos pressupostos normativos acima, buscou-se analisar, por meio das pesquisas constantes dos artigos publicados nos congressos e periódicos nacionais classificados no qualis-capes, a produção acadêmica sobre o uso da informação de custos no setor público a partir de 2010. O foco da pesquisa foi analisar como o tema está sendo discutido, visando encontrar evidências de sua aplicação e resultados.

\section{REFERENCIAL TEÓRICO}

A administração pública é responsável pelo planejamento e controle dos recursos públicos, de forma que atenda às necessidades da sociedade. Meirelles (2014) a define como conjunto de órgãos e serviços do Estado. Então, se a administração pública tem como principal objetivo o bem-estar social da coletividade e oferecer serviços com qualidade, torna-se necessário gerir estes recursos de forma eficiente, para que tal objetivo seja alcançado. Um dos mecanismos de gestão usados pela administração pública é a Contabilidade nela aplicada.

Devido ao processo de convergência às normas internacionais de contabilidade voltadas para o setor público, a contabilidade pública passou a estruturar-se em quatro 
O Uso da Informação de Custos no Setor Público Brasileiro: uma Visão Bibliográfica Simão Pereira da Silva, Melania Leão Botelho, Kenia Fabiana Cota Mendonça, Ronan Pereira

Capobiango

sistemas: o orçamentário, o patrimonial, o de custos e o de compensação. Ressalta-se que um sistema para a informação de custos do setor público tornou-se obrigatório para os fatos ocorridos a partir de 2012.

Assim, a contabilidade de custos poderá atuar como ferramenta essencial no processamento de informações, auxiliando os gestores na tomada de decisão, com a finalidade de obter um feedback em relação aos resultados alcançados (MAUSS \& SOUZA, 2008).

De acordo com Macedo e Klann (2012), foram editadas 10 (dez) Resoluções, regulamentando os principais conceitos e procedimentos a serem adotados acerca da Contabilidade Aplicada ao Setor Público. Tais instrumentos legais estão norteando o grande movimento de mudança de práticas pela qual está passando a Administração Pública no país. A elaboração das normas partiu do princípio de que a Contabilidade Aplicada ao Setor Público não deve se limitar a questões orçamentárias e legais, pois o processo de controle do patrimônio público deve partir do estudo dos fenômenos e transações que o afetam (CFC, 2008a).

Há tempos a implantação de sistemas de custos tem sido induzida pela legislação brasileira: desde a Lei 4.320/64 (BRASIL, 1964), que, em seu art. 99, determina a manutenção de contabilidade especial para determinação dos custos, passando pelo art. 79 do Decreto Lei 200/67 (BRASIL, 1967), que reforça a exigência deque a Contabilidade deverá apurar os custos dos serviços de forma a evidenciar os resultados da gestão, até a Lei Complementar 101 (BRASIL, 2000), ao determinar que a Administração Pública manterá sistema de custos que permita a avaliação e o acompanhamento da gestão orçamentária, financeira e patrimonial. Contudo, a partir de 1. ㅇ de janeiro de 2012, a adoção de um sistema de custos passou a ser obrigatória.

\subsection{A Contabilidade de Custos Aplicada no Setor Público}

No Rio de Janeiro, no ano de 2002, também já havia um sistema de custos implantado. Por meio desse sistema, era possível conhecer o custo por aluno e a formação de indicadores gerenciais, já que mensurava todos os custos da Secretaria 
O Uso da Informação de Custos no Setor Público Brasileiro: uma Visão Bibliográfica Simão Pereira da Silva, Melania Leão Botelho, Kenia Fabiana Cota Mendonça, Ronan Pereira

Capobiango

Municipal de Educação. Em Salvador (BA), o sistema de custeio por absorção foi implantado em três escolas da Secretaria Municipal de Educação no ano de 2003.

No Brasil, no ano de 2005, foi criada a Comissão Interministerial de Custos pela Portaria n. 9 945, com o objetivo de elaborar estudos e diagnósticos para a implantação de sistemas de custos na Administração Pública Federal. Até o ano de 2006, algumas instituições nacionais já possuíam um projeto na área de custos, como, por exemplo: o comando da aeronáutica, as organizações militares prestadoras de serviços, o comando da marinha e do exército e o Banco Central do Brasil. Esta última possui um sistema implantado desde 2003, cuja metodologia de custeio baseia-se no método ABC. Este sistema foi pautado nos princípios de eficiência, eficácia, economicidade e avaliação de desempenho.

A implantação de um sistema de contabilidade de custos é um desafio que se encontra em fase de desenvolvimento. Mas, os debates em relação ao tema vêm se tornando cada vez mais acentuados, à medida que há uma maior preocupação em criar ferramentas que direcionem as decisões e avaliem os resultados da gestão de maneira que melhore o desempenho e a qualidade dos gastos realizados pelos gestores públicos (MAUSS E SOUZA, 2008).

Até 2008, alguns países como Estados Unidos, Austrália, Nova Zelândia, Canadá e Reino Unido já possuíam um sistema de custos implantado no setor público. Nos Estados Unidos, por exemplo, predominou o método de custeio $A B C$ em instituições, como Receita Federal, Força Aérea, Marinha e Exército. Outros países, como Portugal, Inglaterra e Itália, perceberam a necessidade da implantação de uma contabilidade de custos na administração pública e estão movendo esforços para alcançar este objetivo (MAUSS e SOUZA, 2008).

A gestão de custos numa abordagem aplicada ao setor público é bem mais complexa que na abordagem societária. Andrade (2013) ressalta que, enquanto na administração pública a alteração na estrutura organizacional depende de atos normativos do poder Executivo e Legislativo, na administração privada as modificações, no seu ambiente empresarial, dependem da estratégia dos gestores, pois os mesmos não sofrem imposição legal. 
O Uso da Informação de Custos no Setor Público Brasileiro: uma Visão Bibliográfica

Simão Pereira da Silva, Melania Leão Botelho, Kenia Fabiana Cota Mendonça, Ronan Pereira

Capobiango

\subsection{Métodos de Custeio}

Para Hendriksen e Van Breda (1999), por meio do sistema de custos é possível realizar as classificações, análises, avaliações, controles e planejamentos. Ao identificar os gastos com a produção, o sistema de custos transforma-se num importante instrumento de gestão, como fonte primária e básica para a tomada de decisão.

Os métodos de custeios são utilizados pela contabilidade de custos para a operacionalização das atividades de uma empresa, além de registrar informações a respeito dos custos gerados. Porém, buscar um método adequado como ferramenta para apropriar os custos indiretos aos produtos e serviços sempre foi um desafio entre diversos autores.

Para Lima e Castro (2007), os métodos de custeio tradicionais servem de parâmetros para muitas instituições públicas, notadamente as militares e algumas prefeituras pioneiras. No Quadro 1, encontra-se uma comparação entre os três métodos de custeio.

\begin{tabular}{|c|c|c|c|}
\hline $\begin{array}{l}\text { VANTAGENS / } \\
\text { DESVANTAGENS }\end{array}$ & $\begin{array}{c}\text { CUSTEIO } \\
\text { POR ABSORÇÃO }\end{array}$ & $\begin{array}{l}\text { CUSTEIO } \\
\text { VARIÁVEL }\end{array}$ & $\begin{array}{l}\text { CUSTEIO BASEADO } \\
\text { EM ATIVIDADES (ABC) }\end{array}$ \\
\hline Vantagens & $\begin{array}{l}\text { Mais simples; custo / } \\
\text { benefício; baseado no } \\
\text { Princípio da Competência; } \\
\text { adequado à área pública em } \\
\text { razão do tratamento dos } \\
\text { custos fixos, que são } \\
\text { preponderantemente } \\
\text { de longo prazo. }\end{array}$ & $\begin{array}{l}\text { Importância e utilidade } \\
\text { do conceito de } \\
\text { margem } \\
\text { de contribuição; } \\
\text { inexistência de rateio } \\
\text { de custos; fácil } \\
\text { aplicação; custo / } \\
\text { benefício. }\end{array}$ & $\begin{array}{c}\text { Maior qualidade nas } \\
\text { informações de custos } \\
\text { de processos e de } \\
\text { serviços; flexibilidade; maior } \\
\text { sofisticação no tratamento } \\
\text { dos custos indiretos, que } \\
\text { são } \\
\text { preponderantes na área } \\
\text { pública. }\end{array}$ \\
\hline Desvantagens & $\begin{array}{l}\text { Rateio arbitrário dos } \\
\text { custos indiretos e fixos; } \\
\text { precisão não muito } \\
\text { certa, comprometendo a } \\
\text { qualidade da } \\
\text { informação. }\end{array}$ & $\begin{array}{c}\text { Dicotomia: curto prazo } \\
\text { versus } \\
\text { longo prazo, com } \\
\text { ênfase } \\
\text { no curto prazo; } \\
\text { relevância dos custos } \\
\text { indiretos sobre os } \\
\text { diretos. }\end{array}$ & $\begin{array}{l}\text { Oneroso; prazo longo para } \\
\text { implementação e } \\
\text { maturação; complexo no } \\
\text { mapeamento dos } \\
\text { processos e atividades e na } \\
\text { identificação e apropriação } \\
\text { dos custos pelos objetos de } \\
\text { custeio. }\end{array}$ \\
\hline
\end{tabular}

Quadro 1 - Comparação entre as metodologias de custeio Fonte: Adaptado de Martinez e Alves Filho (2010). 
O Uso da Informação de Custos no Setor Público Brasileiro: uma Visão Bibliográfica Simão Pereira da Silva, Melania Leão Botelho, Kenia Fabiana Cota Mendonça, Ronan Pereira

Capobiango

A legislação brasileira não aceita o método de custeio variável para fins tributários e contábeis, embora seja considerado como ferramenta gerencial. Este método não engloba os custos e despesas fixas, mas os custos e despesas diretas, deixando a informação incompleta. Por esta razão e pelas características de os gastos públicos serem predominantemente indiretos, não seria interessante implementar o método de custeio variável no setor público.

O custeio por absorção pode ser implementado com mais facilidade, pois, de acordo com Mauss e Souza (2008), permite ao gestor visualizar os custos totais dos objetos de custeio e verificar qual a influência dos custos indiretos sobre o total dos custos de cada objeto. Porém este método possui como principal ponto negativo a subjetividade das bases de rateio, no que diz respeito à utilização do rateio dos custos indiretos, o que pode comprometer as decisões dos gestores ao distorcerem as informações referentes aos custos dos serviços. Com relação ao custeio $A B C$, este possui certa complexidade ao ser implantado, principalmente na administração pública, pois requer técnicas mais apuradas.

\section{DELINEAMENTO DA PESQUISA}

Quanto aos fins, a pesquisa classifica-se como descritiva. Quanto ao tratamento dos dados, a pesquisa classifica-se em qualitativa, por terem sido analisadas nos artigos as informações que justificassem os dados levantados (MARCONI e LAKATOS, 2008). Para a coleta dos dados, efetuou-se uma busca nos periódicos classificados pela Coordenação de Aperfeiçoamento de Pessoal de Nível Superior (CAPES) para identificação da produção acadêmica publicada nos principais periódicos nacionais que tratam do assunto nos estratos A1 a C. Dos 69 periódicos de Administração e Contabilidade consultados, somente em 15 foram identificados artigos sobre o tema. Em relação aos congressos, foram encontrados artigos em 5 congressos, e todas as publicações de 2010 a 2013 foram analisadas. 
O Uso da Informação de Custos no Setor Público Brasileiro: uma Visão Bibliográfica

Simão Pereira da Silva, Melania Leão Botelho, Kenia Fabiana Cota Mendonça, Ronan Pereira

Capobiango

\section{DESCRIÇÃO DOS DADOS}

São apresentadas as características e os resultados encontrados nos periódicos e congressos nacionais, seguidos dos resultados neles encontrados respectivamente.

\subsection{Periódicos}

A coleta de dados nos 15 periódicos que abordaram o tema resultou em 32 artigos. Na Tabela 1, relacionam-se os periódicos de 2010 a 2013. Percebe-se que a revista na qual houve uma maior incidência foi a Revista de Administração Pública, com 8 artigos publicados, representando 25\% do total dos 32 artigos encontrados. Em segundo lugar, ficou a Revista de Administração Hospitalar e Inovação em Saúde (RAHIS), com 5 artigos, que representou $16 \%$ do total dos artigos.

Tabela 1 - Classificação e quantidade dos Periódicos com artigos sobre custos no setor público de 2010 a 2013

\begin{tabular}{|c|c|c|c|c|}
\hline $\begin{array}{c}\text { Classificação } \\
\text { CAPES }\end{array}$ & Periódicos Nacionais & $\begin{array}{c}\text { № de } \\
\text { artigos }\end{array}$ & $\%$ & $\begin{array}{c}\text { \% } \\
\text { Acumulad } \\
\text { a }\end{array}$ \\
\hline B3 & Cadernos Gestão Pública e Cidadania & 1 & 3,1 & 3,1 \\
\hline B1 & Faces: Revista de Administração & 1 & 3,1 & 6,2 \\
\hline B3 & Perspectivas em Gestão \& Conhecimento & 1 & 3,1 & 9,4 \\
\hline B3 & Revista Ambiente Contábil & 1 & 3,1 & 12,5 \\
\hline B4 & Revista de Contabilidade da UFBA & 1 & 3,1 & 15,6 \\
\hline B3 & Revista de Educação e Pesquisa em Contabilidade & 1 & 3,1 & 18,7 \\
\hline B3 & Revista de Informação Contábil (UFPE) & 1 & 3,1 & 21,9 \\
\hline B4 & Revista Catarinense da Ciência Contábil & 1 & 3,1 & 25,0 \\
\hline B3 & Pensar Contábil & 2 & 6,3 & 31,2 \\
\hline B5 & RBC: Revista Brasileira de Contabilidade & 2 & 6,3 & 37,5 \\
\hline B1 & Revista Contemporânea de Contabilidade & 2 & 6,3 & 43,7 \\
\hline B1 & Revista Universo Contábil & 2 & 6,3 & 50,0 \\
\hline B4 & ABCustos (São Leopoldo, RS) & 3 & 9,4 & 59,4 \\
\hline B3 & Revista de Administração Hospitalar e Inovação em & 5 & 15,6 & 75,0 \\
\hline A2 & Saúde & 8 & 25,0 & 100,0 \\
\hline & Revista de Administração Pública & $\mathbf{3 2}$ & $\mathbf{1 0 0 , 0}$ & \\
\hline
\end{tabular}

Fonte: Dados da pesquisa. 
O Uso da Informação de Custos no Setor Público Brasileiro: uma Visão Bibliográfica Simão Pereira da Silva, Melania Leão Botelho, Kenia Fabiana Cota Mendonça, Ronan Pereira Capobiango

Nota-se também que a maioria dos estratos indicativos foi B3, representado por 12 artigos em 7 periódicos. Não foram encontrados artigos em periódicos de conceito B2 relacionados ao tema, e somente 1 constou em periódico com classificação C.

De acordo com a Tabela 2, nota-se que, entre os períodos analisados, 2010 foi o ano que compreendeu o maior número de artigos publicados, o que representou quase $50 \%$ dos artigos encontrados nos 15 periódicos nacionais. Isso demonstra que o tema desta pesquisa: o uso da informação de custos no setor público, começou e foi fortemente debatido entre os autores naquele ano.

Tabela 2 - Quantidade de artigos encontrados por ano

\begin{tabular}{|c|c|c|}
\hline ANO DAS PUBLICAÇÕES & No DE ARTIGOS ENCONTRADOS & $\%$ \\
\hline 2010 & 14 & 43,8 \\
\hline 2011 & 8 & 25,0 \\
\hline 2012 & 6 & 18,7 \\
\hline 2013 & 4 & 12,5 \\
\hline TOTAL & $\mathbf{3 2}$ & $\mathbf{1 0 0 , 0}$ \\
\hline
\end{tabular}

Fonte: Dados da pesquisa.

Dos autores distribuídos entre os 32 artigos, as publicações em trio (3 autores) foram as que mais se destacaram, com $40 \%$ do total. As pesquisas com quatro autores também tiveram uma representatividade significativa, apresentando $24 \%$ das publicações, conforme demonstrado na Figura 1.

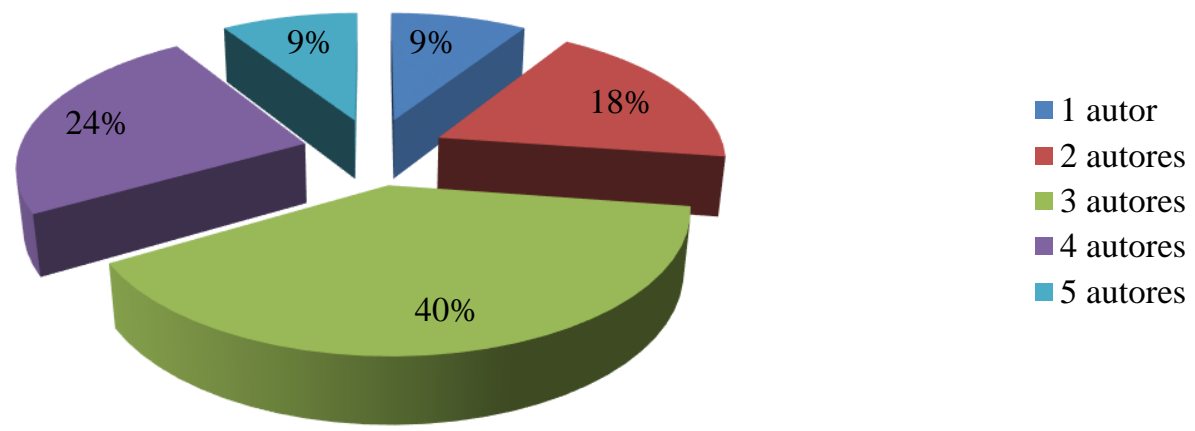

Figura 1 - Quantidade de autores por artigo 
O Uso da Informação de Custos no Setor Público Brasileiro: uma Visão Bibliográfica

Simão Pereira da Silva, Melania Leão Botelho, Kenia Fabiana Cota Mendonça, Ronan Pereira

Capobiango

\subsubsection{Resultados Encontrados nos Periódicos}

Os resultados encontrados nos periódicos sobre o uso da informação de custos são descritos a seguir, com suas principais abordagens e contribuições.

$\mathrm{Na}$ Revista da Associação Brasileira de Custos (ABCustos), foram encontrados três artigos: dois de 2010 e um de 2012.Nota-se, nestes artigos, que não há um método de custeio específico, mas a identificação do método que as necessidades da administração pública reclamam. É o que se percebe de Söthe et al. (2010), ao desenvolver um estudo de caso que teve como objetivo avaliar os custos da Secretaria Municipal de Agricultura e Meio Ambiente de São João do Oeste - SC, por meio da aplicação de um sistema de gestão de custos na administração pública municipal. A pesquisa descritiva, com abordagem quantitativa realizada pelos autores, explanou os três sistemas de custeio (Custeio por Absorção; Custeio Direto ou Variável e Custeio Baseado em Atividades - $A B C$ ), no qual apresentou vantagens e desvantagens de cada um e abordou a legislação pertinente à gestão de custos no setor público. Apesar de afirmarem que ainda não há uma metodologia específica sobre custos no setor público, os autores utilizaram o método de Custeio por Absorção para demonstrar a viabilidade da implantação de um sistema de gestão de custos em nível municipal, pois tal sistema seria capaz de suprir a falta de informações gerenciais. Todavia, só teria avanço se houvesse um controle interno eficaz que facilitaria a apuração dos custos, de modo que garantisse uma boa definição de critérios para a sua distribuição aos produtos ou serviços públicos.

Martinez e Alves Filho (2010) defenderam a ideia de implantação de um sistema mais simples, pelo fato de que o setor público encontra-se ainda em fase de aprendizagem, podendo não suportar sistemas de custeio mais complexos com alto custo de implantação, caso do Custeio ABC. E, por meio de um estudo de caso, eles sugeriram o desenvolvimento de um modelo conceitual de custos para o setor público, cujo objeto de estudo foram três escolas municipais. O objetivo foi chegar aos custos por escola e custo por aluno com a utilização, na sua fase inicial, do custeio por Absorção e, depois, o ABC. As duas metodologias de custos utilizadas demonstraram 
O Uso da Informação de Custos no Setor Público Brasileiro: uma Visão Bibliográfica Simão Pereira da Silva, Melania Leão Botelho, Kenia Fabiana Cota Mendonça, Ronan Pereira

que o custeio por Absorção com departamentalização é o mais indicado no curto prazo, porém, no longo prazo, o Custeio Baseado em Atividades é o mais apropriado.

Carneiro Júnior, Silva e Rocha (2012) demonstraram, através de um estudo de caso, que, desde 2008, já existia o sistema gerencial de custos do exército denominado Siscustos. Esse sistema contou com a ajuda de outros sistemas corporativos, os quais forneciam os dados para apuração dos custos, e utilizou-se de tecnologia da informação para captar os dados de forma que suprisse todo o sistema de custos. $O$ método de custeio mais adequado foi o $A B C$, devido à finalidade de alocar os custos àquelas atividades que utilizavam mais recursos, permitindo assim determinar quais agregavam valor às ações das organizações públicas, tornando possível avaliar a eficiência e eficácia no processo de aplicação desses recursos utilizados.

Em Cadernos Gestão Pública e Cidadania da Fundação Getúlio Vargas, Raupp (2011) fez um estudo de caso exploratório com abordagem qualitativa entre a utilização do método de Custeio por Absorção com o método de Custeio Baseado em Atividades na gestão de custos em uma Câmara do Município de Paulo Lopes (SC). O estudo mostrou que um modelo de gerenciamento de custos é importante no legislativo municipal por servir de auxílio aos gestores na análise dos gastos por centros de custos, de modo que reduza possíveis desperdícios e eliminem atividades desnecessárias, contribuindo assim para o aperfeiçoamento do processo de prestação de contas. As duas propostas de gestão de custos apresentadas por Raupp (2011) utilizaram dois métodos aplicados: o custeio por absorção e o ABC. O Custeio por Absorção teve uma maior facilidade de implantação por considerar critérios de alocação mais simples. $O A B C$, embora fosse viável pelo fato do setor público trabalhar com atividades no momento da elaboração do orçamento, demonstrou um maior custo e implantação mais demorada.

$\mathrm{Na}$ Revista de Administração da universidade FUMEC/FACE, encontrou-se um artigo publicado em 2011 que discute requisitos funcionais de um sistema de informações para gestão de custos no setor público. Trata-se de um estudo de caso desenvolvido por Martinez e Alves Filho (2011) na Secretaria Municipal de Saúde e Educação em que procuraram demonstrar a estrutura do sistema de informações 
O Uso da Informação de Custos no Setor Público Brasileiro: uma Visão Bibliográfica Simão Pereira da Silva, Melania Leão Botelho, Kenia Fabiana Cota Mendonça, Ronan Pereira

voltado para o controle de custos, discutido em artigo de 2010, na Secretaria Municipal de Salvador, pelos mesmos autores. O referencial teórico abordou, de maneira minuciosa, os requisitos necessários para a implantação do sistema de custos em um município. Notou-se que os setores da saúde e da educação requeriam dos gestores públicos atenção especial em relação ao controle de custos, pois as verbas destinadas eram insuficientes, ou os recursos não eram empregados com qualidade. Esse fato evidenciou a necessidade de mensurar os custos desses recursos para contribuir no planejamento e controle dos gastos públicos. O estudo revelou ainda que um sistema de gestão de custos seria possível se houvesse um sistema adequado de controles internos.

No periódico Pensar Contábil do CRC-RJ, dois artigos foram publicados: Raupp (2011) discutiu a proposta de implantação do custeio $A B C$ no legislativo municipal, comparando-o com o método de Custeio por Absorção através de um estudo de caso com abordagem qualitativa, cujos resultados indicaram limitações administrativas para a adoção dos sistemas. Todavia, alguns sistemas implantados apresentaram resultados satisfatórios ao adotarem o Custeio por Absorção; Silva et al. (2010) fizeram um estudo de caso na Marinha do Brasil. Em 1994, a Marinha do Brasil necessitava de um sistema de custos que fosse capaz de mensurar os custos de suas estruturas administrativas. A partir daí, foi criado, pela Alta Administração Naval, o Sistema das Organizações Militares Prestadoras de Serviços (OMPS), com a utilização do Custeio por Absorção. Para Silva et al. (2010), o Custeio por Absorção era mais simples em relação ao Custeio $A B C$, pois este último necessitava de profissionais que soubessem utilizá-lo de forma correta em virtude do grande volume de cálculos que são utilizados. Os resultados demonstraram que o método por Absorção implantado pela Marinha do Brasil estava dando certo, pois atendia a necessidade de informações de custos.

A Revista Perspectivas em Gestão \& Conhecimento, do Departamento de Administração, de Ciência da Informação e de Filosofia da UFPB, registrou 1 artigo referente ao ano de 2013.Por meio de um estudo de caso com observação participante, cujo método adotado caracterizou-se como observacional e monográfico, a pesquisa qualitativa e quantitativa foi desenvolvida em uma Fundação Hospitalar do Estado de 
O Uso da Informação de Custos no Setor Público Brasileiro: uma Visão Bibliográfica Simão Pereira da Silva, Melania Leão Botelho, Kenia Fabiana Cota Mendonça, Ronan Pereira Capobiango

Minas Gerais, a FHEMIG. Para Alemão et al. (2013), a implantação do Sistema de Gestão Estratégica de Custos Hospitalares, em 2007, favoreceu dois grupos importantes: os agentes internos e agentes externos, pois gerou informações de custos de valor. Os autores ainda destacaram que a FHEMIG desenvolveu dois módulos integrados, adotando duas metodologias de custos: o módulo SIGH-Absorção e o módulo SIGH-ABC.

Na Revista de Administração Hospitalar e Inovação em Saúde (RAHIS), foram publicados 5 artigos, todos relacionados à área da saúde. Nascimento e Taborda (2010), em seus estudos, abordaram os sistemas de custos implantados em hospitais públicos administrados por Organizações Sociais de Saúde (OSS), no estado de São Paulo. O estudo de caso foi feito com foco em 18 hospitais públicos administrados por parcerias firmadas entre Secretaria Estadual de São Paulo e Organizações habilitadas com o título OSS. Os resultados demonstraram que os sistemas instalados nos hospitais administrados por OSS estavam contribuindo para a qualidade dos serviços prestados.

Um estudo de caso descritivo realizado por Martins et al. (2010), em uma rede de hospitais públicos, baseou-se na avaliação da implantação de um sistema de Custeio por Absorção na Fundação Hospitalar do Estado de Minas Gerais (FHEMIG), cujos resultados desse sistema mostraram-se positivos para a geração de informações sobre custos hospitalares. Este artigo gerou outro artigo em 2013, que foi publicado em periódico diferente.

Por mais complexa que seja a aplicação do sistema ABC, alguns autores são a favor da utilização deste método em sistemas de custos adotados na saúde pública. Exemplo disso foi a pesquisa descritiva realizada por Gonçalves et al. (2010), que analisaram a aplicabilidade do sistema $A B C$ em um bloco cirúrgico, realizada em um hospital público de Belo Horizonte. Pela análise dos resultados, o método $A B C$ apresentou informações mais concretas, permitiu identificar problemas estruturais relacionados à gestão do hospital, em que diversos setores necessitavam ser reestruturados para que atendessem ao fim desejado, bem como a viabilidade de 
O Uso da Informação de Custos no Setor Público Brasileiro: uma Visão Bibliográfica

Simão Pereira da Silva, Melania Leão Botelho, Kenia Fabiana Cota Mendonça, Ronan Pereira

Capobiango

aplicação do sistema de Custeio $\mathrm{ABC}$, enfatizando o planejamento e o controle como ferramentas primordiais neste processo.

Outra proposta identificada neste periódico foi a de implantação do Sistema de Custos e monitoramento dos Centros Viva Vida. Por meio de um sistema de custos, a coleta das informações contou com dois instrumentos implantados, mostrando-se eficaz na análise da composição dos gastos dos Centros Viva Vida, pois foi possível obter informações mais detalhadas (MARQUES et al. 2010).

Foi publicado também, neste periódico, um estudo exploratório, cuja pesquisa foi realizada em um grupo de hospitais da cidade de Uberlândia - MG. Procurou-se avaliar a utilização das informações referentes aos custos pelos gestores. A pesquisa realizada por meio de questionário, no Hospital de Clínicas de Uberlândia (o único hospital público dos cinco selecionados para o estudo), demonstrou que o funcionamento efetivo de um sistema de custos condiciona-se à padronização dos serviços prestados, processo um pouco complicado por falta de recursos e maior demanda por parte da população (SANTOS et al., 2013).Os resultados encontrados demonstraram o reconhecimento da importância de tais informações, porém a implantação de um sistema de custos geraria gastos muito altos, além de um aumento no nível de controles internos a serem implantados, devido ao fato de que os serviços prestados em um hospital são bastante complexos.

Percebe-se, nesses artigos, que a aplicação de sistema de custos no setor da saúde é mais complexa e que não há um método concebido para tanto, mas a adoção de métodos tradicionais com adaptações à realidade.

$\mathrm{Na}$ Revista Ambiente Contábil, o artigo encontrado identificou, por meio de estudo de caso em Vilhena-RO, os fatores que podem ser empecilhos e dificultar a implantação e o desenvolvimento de um sistema de custos, destacando aspectos relacionados, como a falta de conhecimento do gestor, a falta de interesse desse tipo de controle, a falta de servidores especializados e capacitados e a falta de estudos na área e no planejamento das atividades (CARNEIRO et al., 2013).

$\mathrm{Na}$ Revista de Administração Pública (RAP),8 artigos relacionados ao tema foram encontrados, dos quais destacam-se os comentados a seguir. $\mathrm{O}$ estudo de caso 
O Uso da Informação de Custos no Setor Público Brasileiro: uma Visão Bibliográfica Simão Pereira da Silva, Melania Leão Botelho, Kenia Fabiana Cota Mendonça, Ronan Pereira

realizado na Universidade Federal de Viçosa (UFV), pesquisa de cunho exploratório e bibliográfico, realizada por Magalhães et al. (2010), procurou apurar o custo por aluno no ensino de graduação. Os autores se basearam em fatores que estavam influenciando o comportamento do custo por aluno, como, por exemplo: o número total de funcionários, número de alunos de graduação, número de matrículas nos cursos da graduação, dentre outros. As técnicas utilizadas pelos autores para a seleção destas variáveis ajudaram a alcançar os resultados pretendidos e concluíram que a interferência delas na instituição acarreta um maior ou menor custo por aluno, dependendo da forma como são interpretadas.

Definir um modelo conceitual específico para a administração pública é precipitado, mas os autores Machado e Holanda (2010) publicaram um artigo referente ao Sistema de Informação de Custos do Governo Federal, no qual descreveram um modelo conceitual de custos para o setor público. Os autores reprisam que não é uma tarefa fácil a escolha do método de custeio do setor público, pois há defesa para todos os tipos, não havendo assim limitações quanto à sua escolha, mas a falta de integração dos sistemas estruturantes e de padronização das estruturas organizacionais do governo federal são ainda problemas a serem revistos pelos gestores públicos.

Os autores Bonacim e Araujo (2010) apresentam, por meio de um estudo de caso, a experiência de implantação da metodologia do custeio $A B C$ no Hospital das Clínicas da Faculdade de Medicina de Ribeirão Preto, da Universidade de São Paulo. O hospital não apresentava uma metodologia de mensuração de custos consistente, e havia dificuldades em relação ao financiamento das atividades desenvolvidas. Isso provocou a proposição do sistema de custos por atividades para demonstrar a forma como pode ser mensurado o impacto nos custos assistenciais, de maneira que seja priorizada a transparência e eficiência na gestão dos recursos públicos.

$\mathrm{Na}$ Revista Brasileira de Contabilidade do CFC - Conselho Federal de Contabilidade, foram encontrados dois artigos referentes ao tema. Cavalcante e Hass (2010) demonstraram, através de um estudo de caso no Laboratório de Análises Clínicas em Itapiranga-SC, a apuração dos custos dos exames realizados. Pelo estudo, a determinação dos reais custos de manutenção das atividades por meio do controle 
O Uso da Informação de Custos no Setor Público Brasileiro: uma Visão Bibliográfica Simão Pereira da Silva, Melania Leão Botelho, Kenia Fabiana Cota Mendonça, Ronan Pereira

Capobiango

dos recursos tende a melhorar os serviços prestados à população. Por sua vez, Lima (2011) demonstrou-se favorável à utilização do sistema de custeio $A B C$ ao argumentar que é o ideal para o controle do Resultado Primário na administração pública municipal, pois a necessidade de se implantar um sistema de custeio prende-se ao atendimento da legislação.

Na Revista Catarinense da Ciência Contábil do CRC-SC, Conselho Regional de Contabilidade de Santa Catarina, foi encontrado 1 artigo que utilizou a pesquisa descritiva com o objetivo de verificar a adoção de controles de custos pelas administrações dos municípios catarinenses com mais de 50.000 habitantes. Os dados foram coletados por meio de questionário e respondidos por contadores das prefeituras dos 19 municípios selecionados para a pesquisa. Segundo Roncalio et al. (2012), as áreas de planejamento e contabilidade precisam de mudanças, pois nota-se pouca interação entre elas. São poucas as prefeituras que adotam controle de custos, o que representa uma porcentagem abaixo do mínimo exigido pela lei.

Na Revista Contemporânea de Contabilidade (RCC), os artigos abordados neste periódico possuem metodologias de pesquisas diferentes. $O$ estudo de caso descritivo realizado por Borgert et al. (2010) teve a finalidade de analisar fatores que pudessem interferir no processo de implementação de um sistema de gestão de custos em um hospital público do Rio Grande do Sul. Eles afirmaram que uma dificuldade encontrada na implementação desse sistema é a falta de planejamento, assim como problemas referentes à comunicação entre os setores administrativos e de ortopedia. Por sua vez, ao analisarem publicações de artigos científicos nos anais eletrônicos de três eventos brasileiros a partir da promulgação da Lei de Responsabilidade Fiscal, Carneiro et al. (2012) demonstraram que o Custeio $A B C$ foi o método mais sugerido e discutido entre outros autores.

Na Revista de Contabilidade da UFBA, foi encontrado 1 artigo. Comparar o custo por aluno da UFSC e da UNISUL, utilizando duas metodologias distintas: a dos custos totais da universidade e a dos gastos referentes à educação, foi o que fizeram Soares et al. (2010). Por meio de pesquisas documentais, foi possível concluir que a UFSC possui um custo por aluno maior do que a Unisul. Um dos fatores que justificou é o fato 
O Uso da Informação de Custos no Setor Público Brasileiro: uma Visão Bibliográfica Simão Pereira da Silva, Melania Leão Botelho, Kenia Fabiana Cota Mendonça, Ronan Pereira

Capobiango

de que a educação pública não tem relação direta com custo-benefício, bem como a quantidade expressiva de atividades de pesquisa e extensão realizada pelos professores e a especialização dos mesmos.

$\mathrm{Na}$ Revista de Educação e Pesquisa em Contabilidade, do CFC (Conselho Federal de Contabilidade), foi encontrado 1 artigo. Na pesquisa descritiva, de cunho qualitativo, realizada no estado do Paraná, o objetivo central foi conhecer o nível de entendimento e de adoção quanto aos sistemas de custos por parte dos municípios como passo inicial. De acordo com Franco et al. (2013), aspectos como nível de dificuldades de implantação, grau de conhecimento em relação aos sistemas de custos por parte dos agentes públicos e noções de condições estruturais e financeiras foram analisados no estudo. Os resultados demonstraram que há limitações quanto ao conhecimento pelos agentes, principalmente no que diz respeito à viabilidade orçamentária e financeira da adoção de sistemas de custos, assim como no ambiente para a implantação e nos sistemas de custos implantados e sistemas operacionais.

$\mathrm{Na}$ Revista de Informação Contábil da UFPE,1 artigo foi encontrado.Com o intuito de mostrar um estudo de caso desenvolvido no serviço de transplante hepático do Hospital Universitário Walter Cantídio, da UFC, por meio de pesquisa bibliográfica e documental, Lima et al. (2012) analisaram as implicações em utilizar o custeio-alvo nestes hospitais universitários federais como ferramenta de gestão estratégica de custos. Os resultados mostraram que o custeio-alvo é recomendado, pois ficou evidenciado que ele pode ser aplicado num serviço de um hospital universitário e serve para a redução de custos, de modo que a qualidade do serviço não seja alterada.

$\mathrm{Na}$ Revista Universo Contábil, 2 artigos foram publicados no ano de 2011. Borgert et al. (2011) abordaram um estudo descritivo voltado para custos hospitalares, cujo objetivo foi analisar o comportamento dos custos das instituições administradas pela Secretaria de Estado da Saúde de Santa Catarina. A pesquisa compreendeu os anos 2007 e 2008, e foram selecionados 14 hospitais, e todos utilizavam a metodologia do Custeio por Absorção. Porém, para o estudo, 6 hospitais foram definidos para a amostra por possuírem um sistema de custos totalmente implantado, sendo que os demais estavam ainda em fase de implantação. Os resultados demonstraram que os 
O Uso da Informação de Custos no Setor Público Brasileiro: uma Visão Bibliográfica Simão Pereira da Silva, Melania Leão Botelho, Kenia Fabiana Cota Mendonça, Ronan Pereira

hospitais que tinham uma menor estrutura, no que diz respeito a leitos, funcionários e menor número de atendimento, eram os que apresentavam maiores custos médios de serviços. A análise permitiu concluir que os custos médios de serviços só seriam reduzidos se o número de atendimentos aumentasse. Bitti, Aquino e Cardoso (2011) realizaram uma pesquisa com o objetivo de analisar os estudos publicados em periódicos acadêmicos sobre a aplicação de sistemas de custos no setor público brasileiro, de modo que pudessem evidenciar as contribuições da pesquisa nacional ao tema, assim como métodos empregados e os resultados sistematizados. Porém, os resultados mostraram que os estudos de caso comparados estavam escassos; existiam poucas pesquisas relacionadas ao tema, pois tratava-se de tema recente, que precisava de debate, reflexão e sensibilização para a implantação de sistemas de informação de custos no setor público.

\subsubsection{Análise dos Resultados Encontrados nos Periódicos}

Os resultados evidenciam que um sistema de custos é de suma importância na administração pública com a implantação de, pelo menos, um método de custeio. Alguns autores defendem a utilização do Custeio por Absorção, por ser mais simples, enquanto outros, a utilização do Custeio $A B C$, apesar da sua complexidade e do alto custo de implantação. Por outro lado, o Custeio Variável não foi muito enfatizado nem discutido pelos autores da área.

Nos quatro anos analisados, o ano de 2010 foi o que mais teve publicações. Os estudos em trio e com quatro autores foram os que mais se destacaram. Dos 15 periódicos que fizeram parte da análise, 7 tiveram conceito B3, o que representou quase $50 \%$ do total, e o estudo de caso foi a metodologia de pesquisa mais utilizada nos artigos encontrados.

Alguns autores abordaram os dois métodos de custeio: Absorção e $A B C$ na implantação do sistema de custos; outros abordaram sistemas de custos próprios implantados a partir dos modelos tradicionais com adaptações, e o sistema de acumulação de custos foi citado em apenas um artigo. Os resultados da pesquisa revelaram que, antes mesmo das normatizações do CFC para implantação de sistemas 
O Uso da Informação de Custos no Setor Público Brasileiro: uma Visão Bibliográfica

Simão Pereira da Silva, Melania Leão Botelho, Kenia Fabiana Cota Mendonça, Ronan Pereira

Capobiango

de custos no setor público, já existiam sistemas de informações de custos implantados em alguns órgãos.

\subsection{Congressos}

Foram selecionados 5 congressos da área de Administração e Contabilidade: 0 Congresso USP de Controladoria e Contabilidade, o Congresso Brasileiro de Custos, o Congresso Brasileiro de Contabilidade, o Congresso da Associação Nacional de Programas de Pós-Graduação em Ciências Contábeis (ANPCONT) e o Congresso da Associação Nacional de Pós-Graduação e Pesquisa em Administração (ANPAD). Neste último, foram destacados três eventos relacionados: o Encontro de Administração da Informação (ENADI), o Encontro de Administração Pública (ENAPG) e o Encontro da ANPAD (ENANPAD). Na Figura 2, consta o número de artigos encontrados nos 5 congressos analisados.

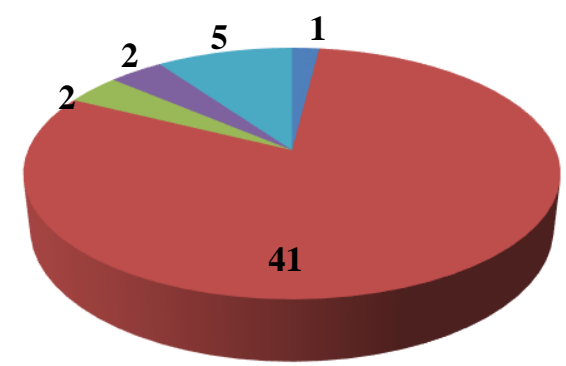

- Congresso USP de Controladoria e Contabilidade

- Congresso Brasileiro de Custos

Congresso Brasileiro de Contabilidade

- ANPCONT

ANPAD

Figura 2 - Quantidade de artigos encontrados nos congressos no período de 2010 a 2013 Fonte: Dados da pesquisa.

\subsubsection{Resultados Encontrados nos Congressos}

Serão descritas, a seguir, as principais contribuições sobre o uso da informação de custos, oferecidas pelos pesquisadores nos congressos. 
O Uso da Informação de Custos no Setor Público Brasileiro: uma Visão Bibliográfica Simão Pereira da Silva, Melania Leão Botelho, Kenia Fabiana Cota Mendonça, Ronan Pereira Capobiango

No Congresso USP de Controladoria e Contabilidade, promovido pela Faculdade de Economia, Administração e Contabilidade da Universidade de São Paulo (FEA/USP), 1 artigos foi encontrado. Destaca-se no artigo encontrado a utilização de informações de custos como suporte à tomada de decisão gerencial em hospitais (SOUZA et al., 2011). Por meio de uma pesquisa com caráter qualitativo e exploratório, analisaram-se cinco hospitais de Belo Horizonte/MG, sendo três de natureza filantrópica, um de natureza privada e outro de natureza pública. A pesquisa consistiu no desenvolvimento de cinco estudos de casos entre os anos 2008 a 2010. Os resultados demonstraram que, apesar de o custeio por absorção ter sido o método utilizado por todos os hospitais em seus sistemas de custos, verificou-se que os sistemas de informações utilizados para gerar as informações de custos nos diversos setores hospitalares apresentaram uma grande heterogeneidade e uma série de deficiências sob a perspectiva dos usuários, o que gerou um baixo índice de satisfação entre os mesmos.

No Congresso Brasileiro de Custos, que acontece anualmente e é o principal evento ligado à área de Custos no Brasil, foram encontrados 41 artigos: destes, 10 artigos em 2010, 9 em 2011, 14 em 2012 e 8 em 2013, cujos estudos discutiram a estrutura administrativa do setor público para recepcionar e aplicar sistemas de custos, bem como analisaram sistemas de custos já implantados. Em síntese, nestes artigos, algumas iniciativas de conscientização da adoção de sistemas de custos estão sendo tomadas à medida que os gestores públicos percebem a importância de implantar um sistema de custos sob a ótica da contabilidade. Segundo Araújo e Silva (2010), experiências como a do Comando da Marinha, das Organizações Militares Prestadoras de Serviços (OMPS) e do Comando da Aeronáutica podem servir de referências para que outras organizações governamentais venham a desenvolver a contabilidade de custos. Há autores que direcionaram o foco de suas pesquisas para a importância da adoção de um sistema de custos; outros, como Davis (2010), propuseram um método de auditoria para avaliar tais sistemas de controle de gestão de custos, a fim de promover melhorias na implantação deles em organizações do setor público. 
O Uso da Informação de Custos no Setor Público Brasileiro: uma Visão Bibliográfica Simão Pereira da Silva, Melania Leão Botelho, Kenia Fabiana Cota Mendonça, Ronan Pereira Capobiango

Nos artigos encontrados, nota-se que o método de Custeio Variável não é aplicado em um sistema de custos pelos gestores. O estudo realizado por Santos et al. (2012) em Organizações Militares Prestadoras de Serviço (OMPS) mostrou que há um distanciamento no que diz respeito às características culturais da OMPS pesquisada em relação ao custeio variável. A centralização das decisões, a ausência de delegação de competência, o modelo organizacional adotado e pouca autonomia proporcionada aos subordinados são fatores que explicam a razão de o custeio variável não ser utilizado em sistemas de custos. Sistemas de custos em instituições públicas, como o Banco Central do Brasil, mostram, no estudo de Faria (2012), que é importante tomar medidas que aumentem a utilização gerencial do sistema e que possibilitem um maior controle dos custos gerados.

No Congresso Brasileiro de Contabilidade que se realiza a cada quatro anos, foram encontrados dois artigos. Propor um modelo de gestão que contribua para a eficiência na aplicação dos recursos públicos destinados ao ensino, à pesquisa, à cultura e à extensão, não é tarefa fácil, porém, Maus et al. (2012), por meio de pesquisa bibliográfica e exploratória de natureza qualitativa, demonstraram, através da utilização do Custeio Baseado em Atividades, objetos e direcionadores de custo, além de direcionadores de recursos e direcionadores de atividades no contexto de uma universidade pública.

A gestão de custos utiliza diferentes modelos para análise da eficiência pública no Brasil e em outros países. Como no Brasil alguns modelos apresentaram resultados satisfatórios, o objetivo da pesquisa realizada por Rosa et al. (2012) foi analisar um modelo de gestão pública por resultados, aplicado no município de Coqueiros do Sul$\mathrm{RS}$, sob a ótica de experiências internacionais. Foram apurados os custos totais das atividades de saúde, usando-se o Custeio $A B C$, cuja escolha do método deveu-se à alta parcela de custos indiretos, e o modelo aplicado foi comparado com experiências internacionais, como as do Canadá e de Portugal. Os resultados identificaram que, nos países pesquisados, não há um modelo informacional como o proposto pela pesquisa, e discutiu a necessidade de o Brasil estar atento às experiências internacionais para poder melhorar seus resultados públicos. 
O Uso da Informação de Custos no Setor Público Brasileiro: uma Visão Bibliográfica Simão Pereira da Silva, Melania Leão Botelho, Kenia Fabiana Cota Mendonça, Ronan Pereira

Capobiango

No Congresso ANPCONT, da Associação Nacional de Programas de PósGraduação em Ciências Contábeis, foram encontrados 2 artigos, tendo um sido publicado em 2010 e outro, em 2012.Temas relacionados à Análise de Custos Baseada em Atividades ( $A B C)$ foram debatidos por Almeida et al. (2010), cujo objetivo era auxiliar a gestão pública municipal na alocação dos recursos e no controle de custos nos serviços públicos educacionais. O custeio na área educacional do município de Alagoinhas- Bahia serviu como fonte de pesquisa para a aplicação do $A B C$ a fim de viabilizar a compreensão dos principais benefícios, dificuldades e resultados de sua utilização. Da aplicação, constatou-se a viabilidade de utilização do $A B C$, tanto para mensurar e controlar custos quanto para análise de processos e atividades do sistema educacional público municipal. Contudo, também foram identificados pontos críticos de gestão como: repetição de trabalhos e gargalos no processo.

Carvalho e Sousa (2012) analisaram se as prefeituras dos municípios gaúchos com população superior a 50.000 habitantes estariam preparadas para a implantação do sistema de custos. O estudo teve como premissa a necessidade que as entidades públicas e privadas têm de atuar com transparência, eficiência e eficácia em suas atividades, tendo nas informações de custos um importante recurso. Os resultados sinalizaram uma carência de recursos destinados à área de custos e precariedade estrutural para o planejamento e controle. Apesar de os gestores municipais se encontrarem cientes quanto à utilidade de um sistema de custos para controle do setor, a pesquisa apontou a necessidade de maior comprometimento e apoio na implantação do sistema. O trabalho revelou que as prefeituras estavam despreparadas para a implantação de sistemas de custos em 2013, inclusive os respondentes da pesquisa apontaram deficiências físicas e limitações humanas para a efetivação do sistema. Havia uma série de medidas que deveriam ser cumpridas para se alcançar a plena efetivação do sistema de custos.

Dos Congressos promovidos pela Associação Nacional de Pós-Graduação e Pesquisa em Administração (ANPAD), foram destacados três eventos: o ENANPAD, o ENADI e o ENAPG. No total de 5 artigos encontrados, discutiram-se assuntos relacionados à comparação do método de Custeio por Absorção em relação ao método 
O Uso da Informação de Custos no Setor Público Brasileiro: uma Visão Bibliográfica Simão Pereira da Silva, Melania Leão Botelho, Kenia Fabiana Cota Mendonça, Ronan Pereira

Capobiango

de Custeio $A B C$, o custo dos projetos de leis do poder Legislativo Federal do Brasil, a gestão de custos no Ensino Básico municipal, o Custeio Baseado em Atividades, com uma análise das pesquisas brasileiras desenvolvidas na primeira década do Século XXI e os custos no setor público, com análise dos artigos posteriores à publicação do estudo $\mathrm{n}^{\circ} 12$ do IFAC.

Os resultados encontrados nestes artigos demonstraram que as áreas da saúde/saneamento/assistência, educação pública e administração municipal merecem uma maior atenção. Quanto à inserção das metodologias de custos nos artigos, a utilização da metodologia do Custeio Baseado em Atividades (ABC) é a que predomina entre os autores, apesar de que o conceito do custo total pode ser adotado como tipologia adequada de custos para o setor público, conforme destacaram Sothe et al. (2012).

Mensurar o resultado econômico por aluno em escolas públicas municipais tem sido uma iniciativa de diversos autores a fim de diminuírem os altos custos com educação e promover a qualidade do ensino. Estudos como o de Barzotto et al. (2010) tem comprovado que municípios com um número maior de alunos possuem um custo médio por aluno muito inferior aos demais, pois conseguem diluir seus gastos fixos, aproveitar melhor a capacidade instalada e obter resultado econômico positivo. O dispositivo constitucional é o grande responsável pelos resultados econômicos do serviço público que afeta negativamente o seu desempenho, pois os gestores, a fim de não incorrerem em penalidade, são obrigados a gastar mais do que realmente precisam para cumprir a legislação.

\subsubsection{Análise dos Resultados Encontrados nos Congressos}

$O$ método de Custeio $A B C$ nos sistemas de custos implantados na administração pública foi muito discutido e adotado entre os autores como o mais adequado. Sistemas já implantados, como o da Marinha, por exemplo, podem servir como referência para que outras organizações governamentais venham a desenvolver seus sistemas de custos. 
O Uso da Informação de Custos no Setor Público Brasileiro: uma Visão Bibliográfica Simão Pereira da Silva, Melania Leão Botelho, Kenia Fabiana Cota Mendonça, Ronan Pereira

As áreas da educação e da saúde foram as mais citadas, e a abordagem parecida com a deste estudo também foi feita por alguns pesquisadores da área. Os resultados encontrados reforçam a caracterização do desafio de aprofundamento das pesquisas e publicações em relação ao tema.

\section{CONSIDERAÇÕES FINAIS}

Dos periódicos das áreas de Administração e Contabilidade pesquisados, em 15 foram encontradas publicações sobre o tema, nos quais 32 artigos foram analisados. Por outro lado, dos 5 congressos selecionados, foram encontrados 51 artigos analisados, com destaque para o Congresso Brasileiro de Custos, com 41 artigos. Em ambos os casos, a pesquisa compreendeu as publicações dos anos 2010 a 2013.

Há uma quantidade relativamente pequena de artigos encontrados nos periódicos, porém robusta no Congresso Brasileiro de Custos. Ressalta-se que, diante da obrigatoriedade recente da utilização do sistema de custos no setor público, o número de artigos é razoável, com relevantes conteúdos que fortalecem o debate acadêmico. Há consideráveis resultados diante da importância do tema para a Contabilidade Aplicada ao Setor Público e para o próprio setor público em modernização.

Percebeu-se que as estruturas administrativas do setor público não estão preparadas ou encontram-se deficientes para implantar um Sistema de Custos, e o que se encontrou foram sistemas de custos implantados com adaptações em áreas específicas.

A implantação de sistemas de custos na educação, em hospitais públicos e em modelos de sistemas de custos militares foi a contribuição mais encontrada e debatida entre os autores, tanto nos periódicos quanto nos congressos, porém ainda não existe uma definição conceitual sobre o sistema de custos para se implantar, mas sistemas tradicionais adaptados em setores específicos.

Os métodos de custeios mais citados pelos autores foram: por Absorção e o Custeio Baseado em Atividades, sendo o $A B C$ o mais debatido entre os autores da 
O Uso da Informação de Custos no Setor Público Brasileiro: uma Visão Bibliográfica

Simão Pereira da Silva, Melania Leão Botelho, Kenia Fabiana Cota Mendonça, Ronan Pereira

Capobiango

área. Esses dois métodos têm sido aplicados, mas com adaptações específicas a cada contexto. A comparação entre eles também esteve presente em alguns estudos realizados e possibilitou conhecer as vantagens e desvantagens de se utilizar um ou outro.

A implantação de uma contabilidade de custos no setor público requer tempo para ser consolidada. Sua exigência legal antecipou-se à preparação administrativa para tanto. Alguns estudos demonstraram que, primeiramente, é necessário desenvolver nos gestores a conscientização da sua importância e a preparação da estrutura gerencial para implantá-lo.

O desenvolvimento de pesquisas sobre Custos no Setor Público é um desafio, e espera-se que os resultados deste estudo possam contribuir no debate. Considera-se que, para a efetivação da Contabilidade de Custos no Setor Público, é de suma importância que seja ampliada a discussão sobre a integração dos métodos a serem adotados para a concepção de sistemas de custos que atendam às regulamentações e às necessidades do setor público em sua complexidade, a partir da utilização de metodologias de custeio que considerem as especificidades, mas também possam ser mais abrangentes.

\section{REFERÊNCIAS}

ANDRADE, Nilton de Aquino. (2013). Contabilidade Pública na Gestão Municipal. (5. ed.). São Paulo: Atlas, 464 p.

BRASIL. Lei 4.320, de 17 de março de 1964. Estatui Normas Gerais de Direito Financeiro para elaboração e controle dos orçamentos e balanços da União, dos Estados, dos Municípios e do Distrito Federal. Diário Oficial [da]República Federativa do Brasil, Brasília, DF, 17 mar. 1964. Disponível em: <http://www.planalto.gov.br/ccivil_03/leis/44320.htm>. Acesso em: 23/jun/2013.

. Lei Complementar no 101, de 04 de maio de 2000. Estabelece normas de finanças públicas voltadas para a responsabilidade na gestão fiscal e dá outras providências. Diário Oficial [da] República Federativa do Brasil, Brasília, DF, 05 maio 2000. Disponível em: <http://www.planalto.gov.br/ccivil_03/leis/lcp/lcp101.htm>. Acesso em: 23/jun/2013. 
O Uso da Informação de Custos no Setor Público Brasileiro: uma Visão Bibliográfica

Simão Pereira da Silva, Melania Leão Botelho, Kenia Fabiana Cota Mendonça, Ronan Pereira

Capobiango

. Decreto Lei no 200, de 25 de fevereiro de 1967. Dispõe sobre a organização da administração federal, estabelece diretrizes para a Reforma Administrativa e dá outras providências. Diário Oficial [da] República Federativa do Brasil, Brasília, DF, 27 fev. 1967. Disponível em: <http://www.planalto.gov.br/ccivil_03/decreto-lei/del0200.htm>. Acesso em: 16/jul/2013.

. Decreto $n^{\circ} 2.829$, de 29 de outubro de 1998. Estabelece normas para a elaboração e execução do Plano Plurianual e dos Orçamentos da União, e dá outras providências. Diário Oficial [da] República Federativa do Brasil, Brasília, DF, 03 out. 1998b. Disponível em: <http://www.planalto.gov.br/ccivil_03/decreto/D2829.htm>. Acesso em: 17/jul/2013.

CONSELHO FEDERAL DE CONTABILIDADE (CFC). Orientações Estratégicas para a Contabilidade Aplicada ao Setor Público no Brasil. Brasília: CFC, 2008a. Disponível em: <http://www3.tesouro.gov.br/contabilidade_governamental/downloads/CCASP_Orientac oes_Estrategicas_Contabilidade.pdf> Acesso em: 20/jul/2013.

. Resolução CFC no 1.129, de 21 de novembro de 2008. Aprova a NBC T 16.2 -

Patrimônio e Sistemas Contábeis. (2008b). Brasília: CFC. Disponível em: <http://www.cfc.org.br>. Acesso em: 15/jul/2013.

. Resolução CFC no 1.366, de 25 de novembro de 2011. Aprova a NBC T 16.11 - Sistema de informação de custos do setor público.Brasília: CFC,2011. Disponível em: <http://www.cfc.org.br>. Acesso em: 15/jul/2013.

HENDRIKSEN, E. S.; BREDA, M. F. V. (1999). Teoria da Contabilidade. São Paulo: Atlas, $552 \mathrm{p}$.

LIMA, Diana Vaz de; CASTRO, Róbison Gonçalves de. (2007). Contabilidade Pública: integrando União, Estados e Municípios (Siafi e Siafem). (3. ed.). São Paulo: Atlas, 248 p.

MACÊDO, Francisca Francivânia Rodrigues Ribeiro; KLANN, Roberto Carlos. (2012). Análise das Normas Brasileiras de Contabilidade Aplicadas ao Setor Público (NBCASP): Um Estudo nas Unidades da Federação do Brasil. In: Associação Nacional de Pós-Graduação e Pesquisa em Administração (ANPAD), 5, Salvador, BA. Anais.. Disponível em: <http://www.anpad.org.br/diversos/trabalhos/EnAPG/enapg_2012/2012_EnAPG398.pdf >. Acesso em: 18/jul/2013. $16 \mathrm{p}$.

MARTINEZ, Antonio L.; ALVES FILHO, Emilio Maltez. (2010). Gestão de Custos no Setor Governamental: Experiência numa Secretaria Municipal de Educação. Revista da Associação Brasileira de Custos (ABC), Brasília, v. 5, n. 3, p. 60-80, set./dez. 
O Uso da Informação de Custos no Setor Público Brasileiro: uma Visão Bibliográfica Simão Pereira da Silva, Melania Leão Botelho, Kenia Fabiana Cota Mendonça, Ronan Pereira Capobiango

Disponível em: <http://www.unisinos.br/abcustos/_pdf/193.pdf>. Acesso em: 27/abr/ 2014. 24 p.

MATIAS-PEREIRA, José. (2012). Manual de Gestão Pública Contemporânea. São Paulo: (4. ed.). Atlas, $328 \mathrm{p}$.

MARCONI, Marina de Andrade; LAKATOS, Eva Maria. (2008). Técnicas de pesquisa: planejamento e execução de pesquisas, amostragens, e técnicas de pesquisas, elaboração, análise e interpretação de dados. (7. ed.). São Paulo: Atlas, 296 p.

MAUSS, César Volnei; SOUZA, Marcos Antonio de. (2008). Gestão de Custos aplicada ao setor público: Modelo para Mensuração e Análise da Eficiência e Eficácia Governamental. São Paulo: Atlas, 210 p.

MEIRELLES, Hely Lopes. (2014). Direito Administrativo Brasileiro. 40. ed. São Paulo: Malleiros, $926 \mathrm{p}$.

VERGARA, Sylvia Constant. (2014). Projetos e relatórios de pesquisa em administração. 15. ed. São Paulo: Atlas, 104 p.

Data de Submissão: 25/06/2015

Data de Aceite: 06/09/2016 